'Unidad de Prevención Cardiovascular y Rehabilitación Cardiaca, Hospital DIPRECA. Santiago, Chile.

${ }^{2}$ Unidad de Cardiología, Servicio de Medicina, Hospital de Carabineros. Santiago, Chile. ${ }^{3}$ Servicio de Cardiología, Hospital DIPRECA. Santiago, Chile.

${ }^{4}$ Medicina Preventiva Carabineros de Chile, Dirección de Previsión Carabineros de Chile. Chile. ${ }^{5}$ Departamento de Matemáticas, Facultad de Ciencias, Universidad de la Serena. La Serena, Chile.

Recibido el 22 de marzo de 2015 , aceptado el 16 de octubre de 2015.

Correspondencia a: Dra. Paola Varleta Cardióloga, Unidad de Prevención Cardiovascular y Rehabilitación Cardiaca, División de Cardiología y Cirugía Cardiaca. Hospital de la Dirección de Previsión de Carabineros de Chile. Vital Apoquindo $1200,2^{\circ}$ piso. Las, Condes, Santiago, Chile. Teléfono: 5629517274 pvarleta@manquehue.net

\section{Ateroesclerosis subclínica en población de riesgo cardiovascular bajo y moderado por Framingham chileno}

\author{
PAOLA VARLETA ${ }^{1,2}$, ROBERTO CONCEPCIÓN ${ }^{2,3}$, PATRICIO JULIO ${ }^{3}$, \\ HÉCTOR CASANOVA ${ }^{4}$, CARLOS NAVARRETE 5 .
}

\section{Subclinical atherosclerosis among Chilean subjects classified as having a low cardiovascular Framingham risk}

Background: Framingham risk score (FRS) has limitations and can underestimate risk. Carotid ultrasound to measure intima media thickness or plaques is recommended for cardiovascular risk assessment. Aim: To determine the prevalence of subclinical atherosclerosis in asymptomatic subjects classified as low and intermediate risk. Material and Methods: Cross-sectional study performed in subjects without cardiovascular disease. Cardiovascular risk was estimated using the Chilean FRS and the General Cardiovascular Disease FRS. Carotid ultrasound was performed in all subjects. We defined subclinical atherosclerosis as the presence of carotid plaque or intima media thickness greater than 75th percentile by gender and age. Results: We studied 203 subjects aged $47.2 \pm 9.6$ years ( $54 \%$ males). Mean intima media thickness was $0.62 \pm 0.1$ $\mathrm{mm}$. An abnormal value was detected in 68 subjects (33\%) and carotid plaques in 32 subjects (15.7\%). Based on Chilean FRS, 96\% were considered at low risk, $4 \%$ at intermediate, and none at high risk. In the low risk group the prevalence of abnormal intima media thickness and plaques was 40 and 14\% respectively. Presence of plaques was more common in women than men (23 and 7\% respectively, $p<0.01$ ). According to the General Cardiovascular Disease FRS, 23\% were at low, 39\% at low intermediate and $28 \%$ at high intermediate risk. In the low risk group the prevalence of an abnormal intima media thickness was $34 \%$ and no subject had plaques. Conclusions: Carotid plaques were detected in Chilean subjects classified as having a low FRS risk. The underestimation of risk was higher in Chilean women.

(Rev Med Chile 2016; 144: 30-38)

Key words: Atherosclerosiss; Carotid stenosis; Risk assessment; Risk factors.
L a estimación de riesgo cardiovascular en población asintomática es un desafío. Para cuantificarlo se han utilizado tablas de riesgo cardiovascular basadas en estudios epidemiológi$\cos ^{1-3}$. La más utilizada es la puntuación de riesgo de Framingham (PRF), que estima riesgo coronario a 10 años $^{4}$, pero subestimaría riesgo en mujeres ${ }^{5}$.

Por lo anterior, guías internacionales recomiendan utilizar exámenes de imágenes o bio- marcadores para evaluar riesgo cardiovascular en población asintomática de riesgo intermedio o moderado ${ }^{6}$. Dentro de los exámenes de imágenes, el grosor íntima-media-carotídea (GIM) ha sido utilizado por representar estadios subclínicos de ateroesclerosis y ser predictor de eventos cardio y cerebrovasculares $^{7-10}$.

La habilidad de reclasificar con ecografía carotídea ha sido validada en estudios con se- 
guimiento de eventos ${ }^{11,12}$. Cohortes como la del ARIC y Framingham han demostrado la utilidad de detectar placa carotídea para reclasificar población de riesgo moderado, utilizando la PRF para enfermedad cardiovascular general $(\mathrm{CVG})^{13-15}$. Esta puntuación difiere a la PRF adaptada a población chilena (PRF-AdCh), que predice riesgo coronario a 10 años ${ }^{16}$.

Desconocemos si la ecografía carotídea aporta información adicional a la estimación de riesgo en población chilena. Si un sujeto asintomático presenta ateroesclerosis subclínica, su riesgo cardiovascular es mayor y el clínico debería insistir en una terapia intensiva. De este modo, el objetivo del presente estudio fue determinar la prevalencia de aterosclerosis subclínica en sujetos asintomáticos catalogados de riesgo bajo y moderado a través de PRF-AdCh y comparar con PRF-CVG, estimando reclasificación a un riesgo mayor.

\section{Pacientes y Método}

Estudio de corte transversal en sujetos referidos a chequeo cardiovascular entre agosto de 2009 a abril de 2012. Todos firmaron consentimiento informado para su participación, aprobado por comité de ética local.

Se incluyeron sujetos asintomáticos en lo cardiovascular, sin historia de angina ni disnea, entre $\geq 30$ y $<70$ años. Se excluyeron aquellos con antecedente de enfermedad ateroesclerótica (coronaria, cerebrovascular y/o vascular), y/o dislipidemia familiar genética.

\section{Recolección de datos}

Se realizó encuesta sobre antecedentes demográficos, médicos y hábitos. Se realizaron mediciones antropométricas. La presión arterial se midió sentado con manómetro aneroide en reposo, bajo normas establecidas, promediando tres tomas ${ }^{17}$.

Los exámenes sanguíneos se realizaron en ayuno de $12 \mathrm{~h}$. El análisis del colesterol total, colesterol HDL y triglicéridos se realizó con método enzimático colorimétrico en equipo cobas c501 sistema Roche/Hitachi. Se calculó colesterol LDL a través de fórmula de Friedewald. La glicemia se determinó con método enzimático de hexoquinasa en equipo Cobas c501.

Se consideró hipertenso a todo sujeto con diagnóstico de hipertensión arterial bajo definición de JNC7, con o sin fármacos y/o con pre- sión arterial $\geq 140 / 90 \mathrm{mmHg}^{17}$. Dislipidemia se definió ante diagnóstico de hipercolesterolemia, independiente de estar con farmacoterapia, y/o si el sujeto presentaba colesterol total $\geq 200 \mathrm{mg} /$ $\mathrm{dL}$, y/o colesterol LDL $\geq 130 \mathrm{mg} / \mathrm{dL}$. Se consideró diabético si el sujeto refería diagnóstico previo con o sin tratamiento, o ante glicemia de ayuno $>126$ $\mathrm{mg} / \mathrm{L}$. Se definió tabáquico activo todo sujeto fumador diario de cigarros durante el último mes. Se definió obesidad como un IMC $\geq 30$ y síndrome metabólico por ATPIII ${ }^{18}$.

\section{Puntuación de riesgo de Framingham}

Se calculó PRF-AdCh a través de planilla Excel de su sitio web ${ }^{19}$. Esta PRF evalúa el riesgo de infarto miocárdico o muerte de causa coronaria a 10 años, incorporando las variables edad, colesterol total, colesterol HDL, tabaquismo, diabetes, presión sistólica y diastólica ${ }^{16}$. Los sujetos se categorizaron en riesgo bajo $<5 \%$, moderado 5 a $9 \%$, alto 10 a $19 \%$ y muy alto $\geq 20 \%$ según la probabilidad de presentar eventos.

También a cada sujeto se le estimó PRF-CVG (coronaria, cerebrovascular, vascular periférica e insuficiencia cardiaca) a 10 años ${ }^{15}$, utilizando fórmula Excel del sitio web del Framingham Heart Study ${ }^{20}$. Los sujetos se categorizaron en riesgo bajo $<5 \%$, moderado bajo 5 a $10 \%$, moderado alto $>10$ a $<20 \%$ y alto $\geq 20 \%$ de probabilidad de presentar eventos.

\section{Medición por ultrasonido}

El GIM se midió con ultrasonido modo B de alta resolución en equipo Vivid-7 GE utilizando transductor lineal vascular de $7 \mathrm{MHz}$. Las mediciones se realizaron a nivel de pared posterior de carótida común en segmento de $1 \mathrm{~cm}$ proximal al bulbo carotídeo durante la diástole ${ }^{21}$. La medición se realizó con programa de detección de bordes automático para GIM, obteniéndose valor medio. Se posicionó transductor utilizando arco de Meijer ${ }^{22,23}$. Se realizaron 6 mediciones en distintos ángulos preestablecidos. Las mediciones fueron realizadas por un sonografista cardiólogo.

Para el análisis del estudio consideramos ateroesclerosis subclínica la presencia de GIM elevado y/o presencia de placa carotídea, siendo GIM elevado un valor medio superioral percentil 75 para población chilena según edad y sexo ${ }^{24}$. Se consideró placa a aquella protrusión al lumen mayor a $50 \%$ del GIM adyacente o GIM $>1,5 \mathrm{~mm}^{21}$. 


\section{Análisis estadístico}

Para el análisis de los datos se utilizó programa estadístico R 3.0.1 $1^{25}$. Los valores se presentan como media \pm DS para variables continuas y como porcentaje para variables discretas. Para comparar grupos independientes, se utilizó test exacto de Fisher para variables categóricas dicotómicas, y la prueba $t$ de student para variables continuas, además de modelos de regresión logística, ANOVA y regresión lineal. Para la asociación entre variables continuas se ocupó correlación de Pearson y modelos de regresión lineal. Se consideró $\mathrm{p}<0,05$ para resultados estadísticamente significativos, e intervalos de confianza de $95 \%$.

\section{Resultados}

Se estudiaron 203 sujetos, edad $\overline{\mathrm{X}}$ 47,2 \pm 9,6 años, $54 \%$ hombres. Las características demográficas y clínicas se detallan en la Tabla 1. Destaca alta prevalencia de factores de riesgo: $56 \%$ hipertensos y $61 \%$ dislipidémicos. Las mujeres presentaban mayor edad $(\mathrm{p}<0,0001)$ y prevalencia de hipertensión (p: 0,001). Los hombres presentaban mayor cintura abdominal ( $\mathrm{p}<0,001)$, y niveles más bajos de colesterol HDL $(\mathrm{p}<0,0001)$.

\section{Hallazgos ecocardiográficos}

El valor promedio del GIM en la población fue de 0,62 $\pm 0,1 \mathrm{~mm}$. El GIM se asoció con edad $(\mathrm{p}<0,0001)$, PAS $(\mathrm{p}<0001)$, LDL $(\mathrm{p}<0,003)$, colesterol total $(\mathrm{p}=0,02)$, HTA $(\mathrm{p}<0,001)$, tratamiento anti hipertensivo $(\mathrm{p}<0,001)$, tabaquismo $(\mathrm{p}<0,04)$, síndrome metabólico $(\mathrm{p}<0,001)$ y dislipidemia $(\mathrm{p}<0,001)$.

Se detectó GIM elevado en 68 sujetos (33\%). En la Tabla 2 se presenta el valor promedio del GIM según categoría de riesgo. Se observó aumento gradual del GIM a mayor categoría de riesgo, siendo este incremento significativo con la PRFCVG $(\mathrm{p}<0,0001)$.

Se detectó placa en 32 sujetos (15\%); siendo

Tabla 1. Características demográficas, antropométricas y clínicas de la población estudiada total y por sexo

\begin{tabular}{|c|c|c|c|c|}
\hline & Hombres (n: 108) & Mujeres (n: 95) & Total (n: 203) & $\mathbf{p}$ \\
\hline Edad (años) & $43,5 \pm 7,8$ & $51,3 \pm 9,9$ & $47,2 \pm 9,6$ & $<0,0001$ \\
\hline PAS (mm Hg) & $126 \pm 12$ & $130 \pm 14,3$ & $127,8 \pm 13,2$ & 0,04 \\
\hline PAD (mm Hg) & $82,3 \pm 8,3$ & $81,8 \pm 8,8$ & $82,1 \pm 8,5$ & NS \\
\hline Col Total (mg/dL) & $209 \pm 43$ & $211 \pm 36$ & $210 \pm 40$ & NS \\
\hline Col LDL (mg/dL) & $127,9 \pm 38$ & $126,9 \pm 30$ & $127,4 \pm 34$ & NS \\
\hline Col HDL (mg/dL) & $44,5 \pm 10$ & $52,4 \pm 12,7$ & $48 \pm 12$ & $<0,0001$ \\
\hline Col no HDL (mg/dl) & $164,8 \pm 41,1$ & $159,3 \pm 35,8$ & $162 \pm 38$ & NS \\
\hline Triglicérido (mg/dl) & $191 \pm 127$ & $158,9 \pm 87$ & $176 \pm 110$ & $<0,04$ \\
\hline Glicemia ayuno & $95,5 \pm 18,7$ & $96,1 \pm 14,3$ & $95,8 \pm 16,7$ & NS \\
\hline Cintura abdominal & $100,9 \pm 8,6$ & $92,5 \pm 9,4$ & $97 \pm 9,9$ & $<0,001$ \\
\hline IMC & $29,2 \pm 3,6$ & $28,6 \pm 3,9$ & $28,9 \pm 3,7$ & NS \\
\hline Obesidad (\%) & 41,6 & 29,5 & 35,9 & NS \\
\hline HTA (\%) & 45,3 & 69,4 & 56,6 & $<0,001$ \\
\hline Trat. HTA (\%) & 33,3 & 61 & 46,3 & $<0,001$ \\
\hline Diabetes (\%) & 6,4 & 7,3 & 6,9 & NS \\
\hline Tabaquismo (\%) & 31,4 & 22,1 & 27 & NS \\
\hline S. Metabólico (\%) & 36,1 & 47,3 & 41,3 & NS \\
\hline Dislipidemia (\%) & 57,7 & 66,6 & 61,8 & NS \\
\hline PRF AdCh (\%) & $2,2 \pm 1,1$ & $1,8 \pm 0,9$ & $2 \pm 1,1$ & $<0,01$ \\
\hline PRF CVG (\%) & $10,2 \pm 7,7$ & $9,1 \pm 6,5$ & $9,7 \pm 7,2$ & NS \\
\hline
\end{tabular}

Información expresada como porcentaje o promedio \pm desviación estándar. 


\section{Tabla 2. Valor promedio del GIM de la población total por categoría de riesgo aplicando puntuación de Framingham adaptada a Chile o cardiovascular general}

\begin{tabular}{|ccc|}
$\begin{array}{l}\text { Framingham } \\
\text { Categoría } \\
\text { de riesgo }\end{array}$ & $\begin{array}{c}\text { Adaptado Chile } \\
\text { GIM }\end{array}$ & $\begin{array}{c}\text { CV General } \\
\text { GIM }\end{array}$ \\
\hline Bajo & $0,63 \pm 0,12 \mathrm{~mm}$ & $0,57 \pm 0,08 \mathrm{~mm}^{*}$ \\
\hline Moderado & $0,65 \pm 0,09 \mathrm{~mm}$ & $0,63 \pm 0,08 \mathrm{~mm}^{*}$ \\
\hline Alto & - & $0,64 \pm 0,11 \mathrm{~mm}^{*}$ \\
\hline Muy alto & - & $0,76 \pm 0,21 \mathrm{~mm}^{*}$ \\
\hline
\end{tabular}

Información expresada en promedio \pm desviación estándar ${ }^{*} p<0,0001$.

más prevalente en mujeres (24\%) que en hombres $(8 \%)(\mathrm{p}<0,003)$. La presencia de placa se asoció significativamente con hipertensión (OR: 3,96; $1,57-10,32 ; \mathrm{p}<0,003)$ tratamiento antihipertensivo (OR: 3,57; 1,49-8,56; $\mathrm{p}<0,001$ ), y dislipidemia (OR: 2,7; 1,01-7,13; $<\mathrm{p}<0,03$ ).

\section{Puntuación de Framingham adaptado a Chile}

El valor promedio de la PRF-AdCh en la población total fue de $2 \pm 1,1$, siendo de $1,8 \pm 0,9$ en mujeres, menor que en hombres, $2,2 \pm 1$ (p: 0,01). El $96 \%$ de la población fue clasificada en riesgo bajo, y ningún sujeto calificó en riesgo alto (Figura 1).

Los hallazgos ecográficos en grupos de riesgo se muestran en la Figura 2. Destacan los hallazgos en el grupo de "bajo" riesgo, con presencia de ateroesclerosis subclínica en $40 \%$ y placa en $14 \%$. La prevalencia de ateroesclerosis subclínica fue de
$29 \%$ en hombres y de $60 \%$ en mujeres ( $\mathrm{p}<0,003$ ) y de placa de $7 \%$ versus $24 \%$ respectivamente $(\mathrm{p}<0,003)$.

No hubo diferencia entre la PRF-AdCh promedio entre sujetos con y sin placa $(2,4 \pm 1,1$ vs $2,0 \pm 1,1 ; \mathrm{NS})$.

\section{Puntuación de Framingham CVG}

El valor promedio de la PRF-CVG fue de 9,7 $\pm 7(9,1 \pm 6$ en mujeres vs $10,2 \pm 7$ en hombres, $\mathrm{p}=\mathrm{NS})$. Sí se observó diferencia significativa entre el valor promedio de la PRF-CVG en sujetos con presencia de placa $(12,4 \pm 5)$ vs sin placa $(9,2 \pm 7)$, (p: 0,005).

La distribución de riesgo de la población fue la siguiente: $23 \%$ de los sujetos clasificó en riesgo bajo (20\% hombres y $26 \%$ mujeres), $39 \%$ en moderado bajo, $29 \%$ en moderado alto y $9 \%$ en alto; muy diferente a la distribución con puntuación chilena (Figura 1).

La prevalencia de placa y ateroesclerosis se detalla en la Figura 2. En el grupo de bajo riesgo ningún sujeto presentó placa, si se observó 34\% de ateroesclerosis subclínica por GIM elevado. La presencia de placa fue menor en hombres, en 7,5\% y $11 \%$ en grupos de riesgo moderado bajo y alto, versus en $28 \%$ y $45 \%$ en mujeres respectivamente (Tabla y Figura 3 ).

Finalmente se realizó análisis del GIM según PRF confrontando grupos. Se observó que dentro de la categoría de riesgo bajo por PRF-AdCh había sujetos de moderado a alto riesgo según PRF-CVG, y éstos sí se correlacionaron positivamente al GIM $(\mathrm{p}<0,0001)$ (Figura 4).

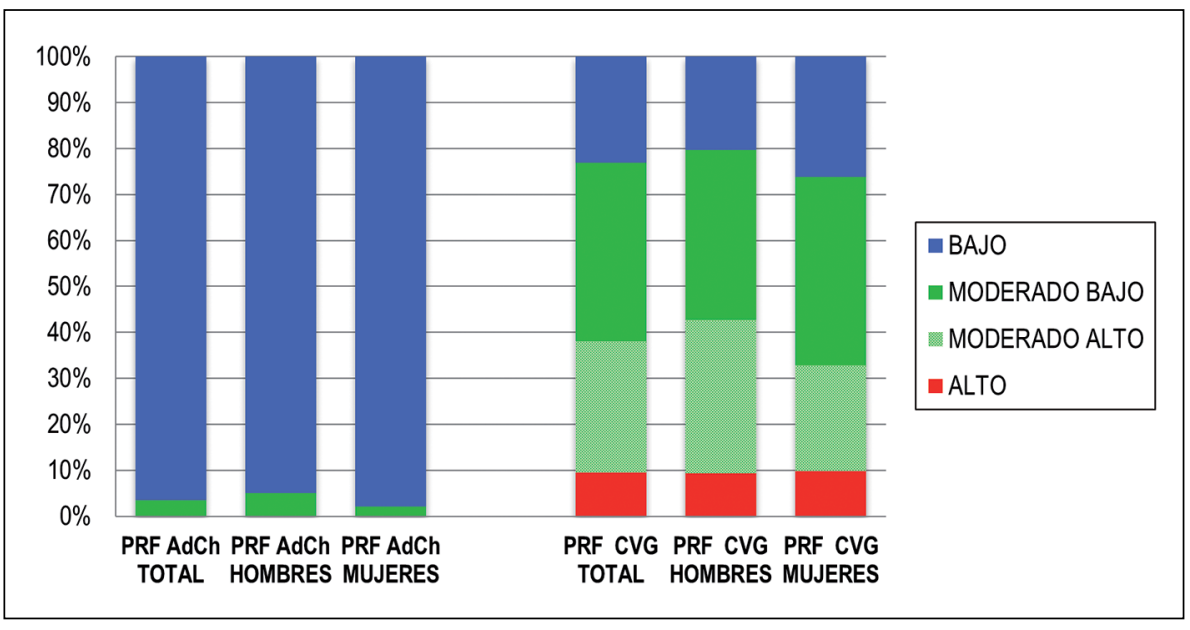

Figura 1. Distribución de riesgo cardiovascular de la población total, hombres y mujeres según puntuación de Framingham aplicada expresado en \%. 


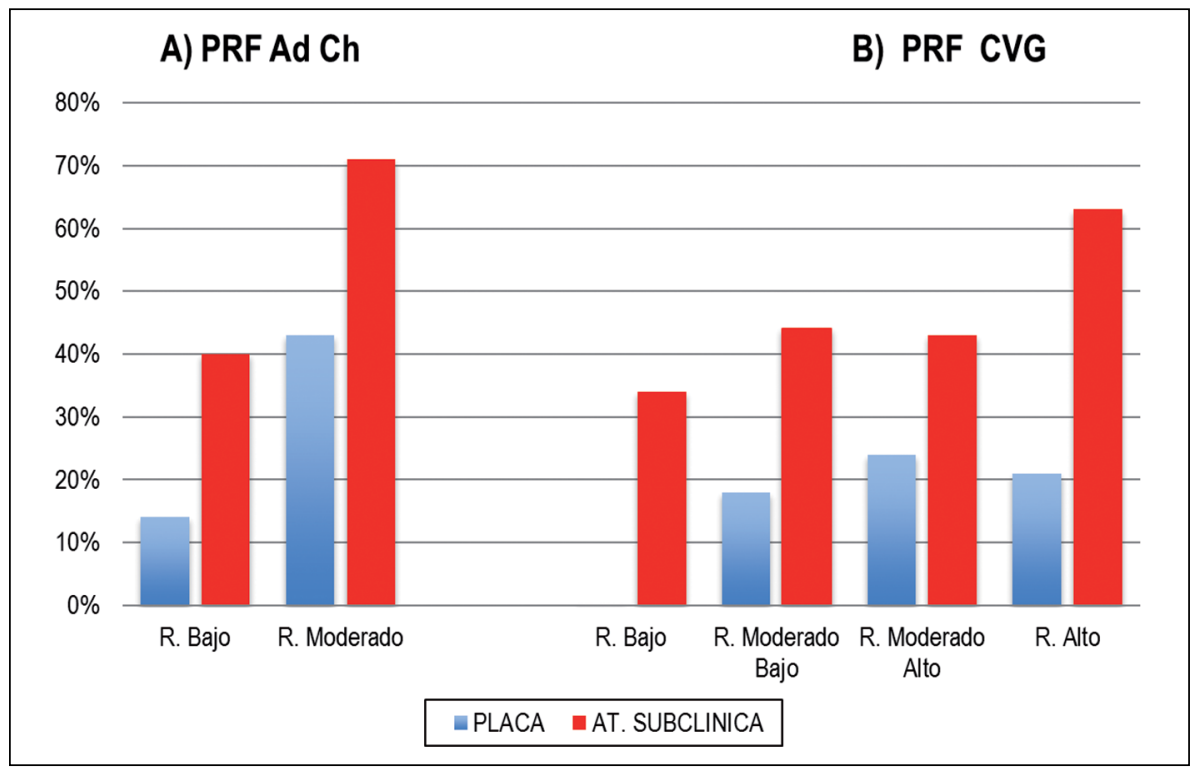

Figura 2. Prevalencia en población total de placa carotídea y ateroesclerosis subclínica en cada categoría de riesgo según PRF aplicada: A) Puntuación de Framingham chileno y B) Framingham cardiovascular general (los valores se expresan $\%$ del total de cada categoría).

Tabla 3. Prevalencia de placa carotídea en mujeres y hombres según categoría de riesgo por puntuación de Framingham Ad Ch y CVG

\begin{tabular}{|c|c|c|c|c|c|c|}
\hline & \multicolumn{2}{|c|}{ PRF Ad Ch } & \multicolumn{4}{|c|}{ PRF CVG } \\
\hline & R. bajo & R. moderado & R. bajo & R. mod bajo & R. mod alto & R. alto \\
\hline & n $\quad(\%)$ & n $\quad(\%)$ & n $\quad(\%)$ & n $\quad(\%)$ & n $\quad(\%)$ & n $\quad(\%)$ \\
\hline Mujeres (n: 95) & $22 / 93(24 \%)$ & $1 / 2 \quad(50 \%)$ & $0 / 25 \quad(0 \%)$ & $11 / 39$ (28\%) & $10 / 22$ (45\%) & $2 / 9 \quad(22 \%)$ \\
\hline Hombres (n: 108) & $7 / 103 \quad(7 \%)$ & $2 / 5 \quad(40 \%)$ & $0 / 22 \quad(0 \%)$ & $3 / 40 \quad(7,5 \%)$ & $4 / 36$ (11\%) & $2 / 10 \quad(20 \%)$ \\
\hline
\end{tabular}

Los valores se expresan en número de sujetos portadores del total de sujetos en cada categoría y en \%.

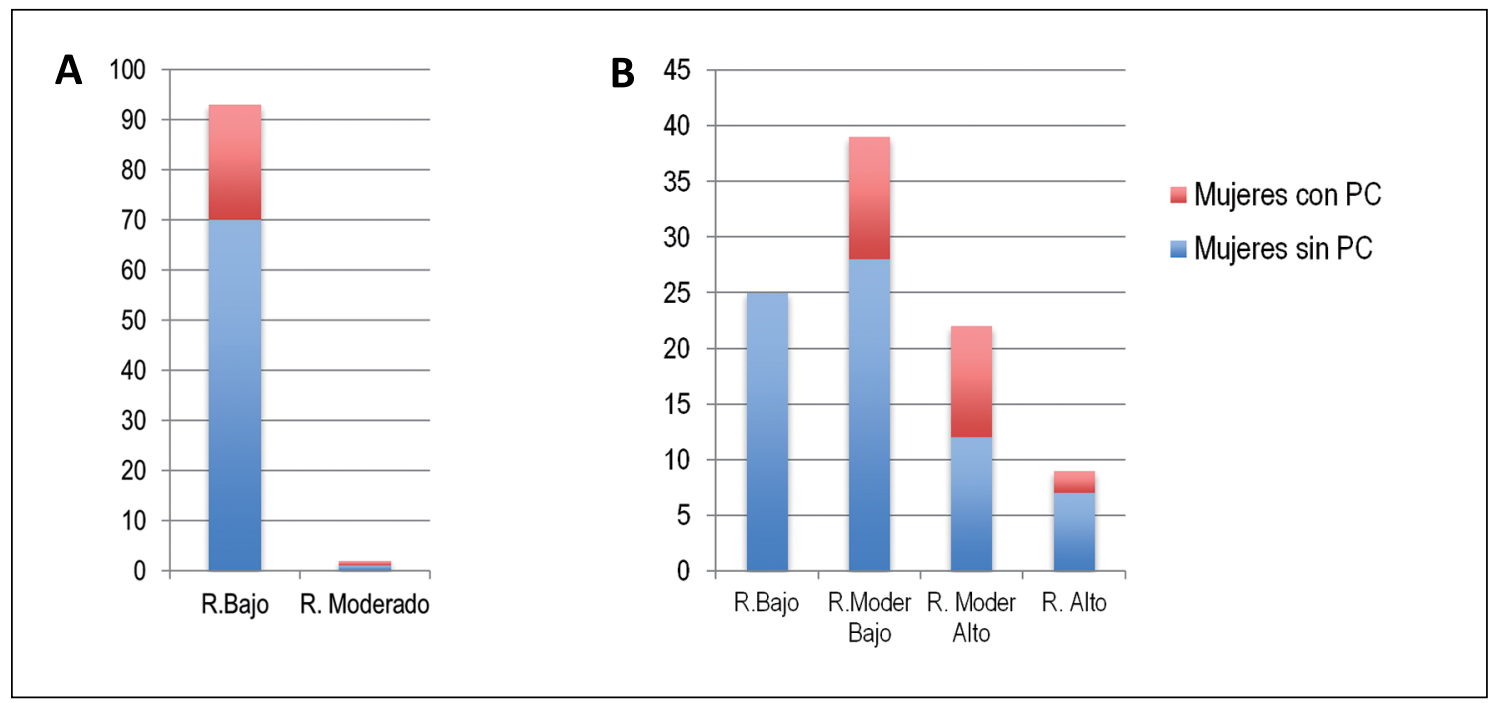

Figura 3. Gráfica que ilustra Número de mujeres con placa carotídea distribuidas según categoría de riesgo por $\mathbf{A}$ ) PRF Ad-Ch y por B) PRF CVG. 


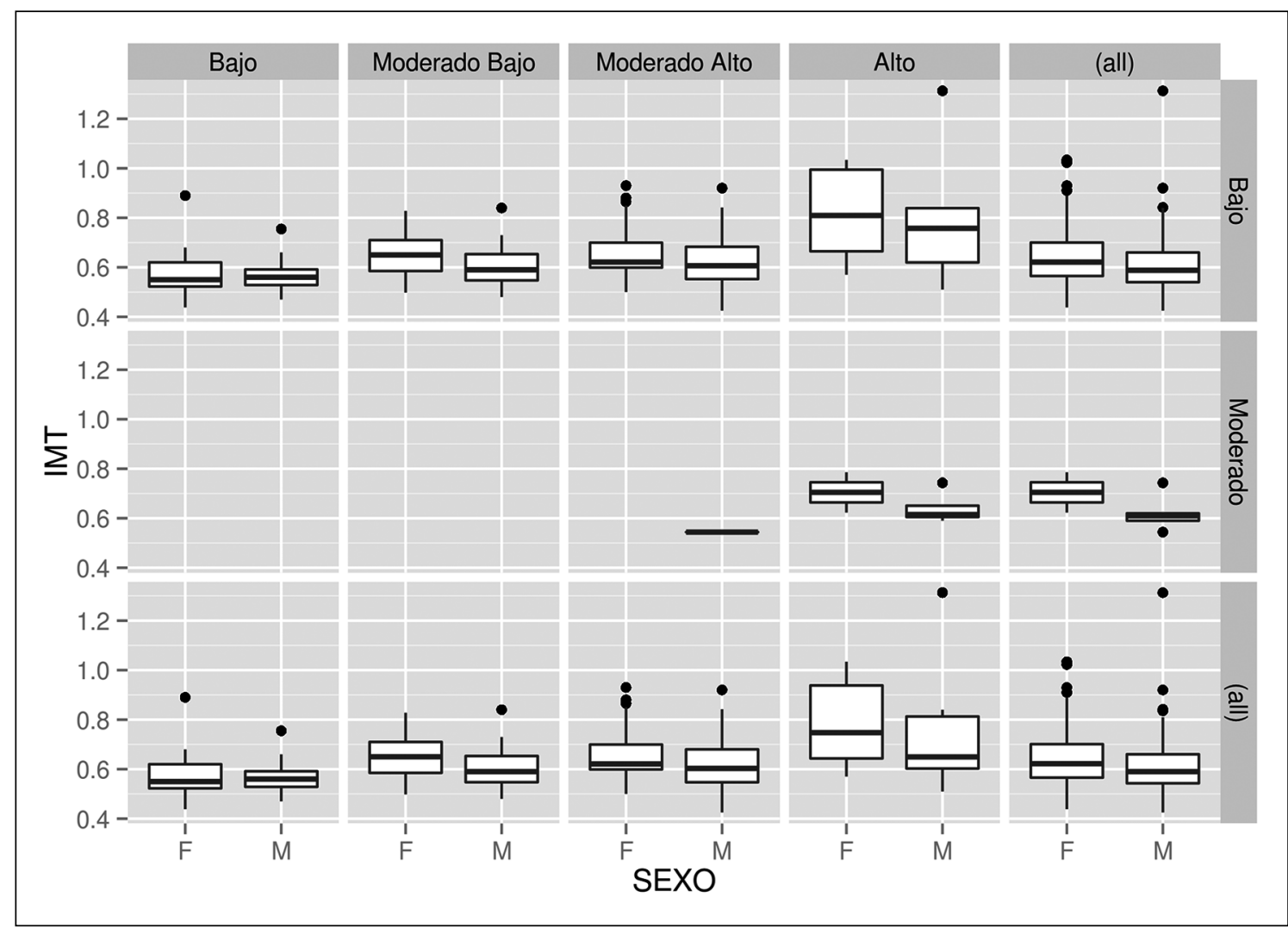

Figura 4. Gráfico que muestra la distribución del GIM según PRF. En el eje X (fila 3) se presenta la clasificación según el PRF CVG, y en el eje Y (columna der) según PRF Ad Ch. Cada celda muestra la distribución del GIM para cada combinación de categorías de ambos puntajes según sexo (gráfica caja y bigote, línea central traduce la mediana, la caja el rango intercuartil y los extremos las observaciones mínimas y máximas).

\section{Discusión}

Este estudio es el primero realizado en población chilena que estratifica a sujetos con PRF-AdCh y ultrasonido carotídeo. En guías internacionales se sugiere recurrir a imágenes o biomarcadores para optimizar la estratificación de riesgo del sujeto sin enfermedad cardiovascular ${ }^{6}$. Esta recomendación está dirigida al grupo de riesgo moderado. Las técnicas de imágenes recomendadas son score de calcio coronario por tomografía computada, y ultrasonido carotídeo con medición del GIM y detección de placa carotídea. La ventaja de este último es la ausencia de radiación y menor costo. Recordamos que en el estudio ARIC, fue la detección de placa por sobre GIM el que objetivó mejor predicción de riesgo cardiovascular, y mayor fortaleza de reclasificación ${ }^{13}$.
Adicional a las puntuaciones de riesgo, es razonable identificar a los sujetos portadores de ateroesclerosis, considerando que la presencia de placa carotídea se ha asociado a mayor tasa de eventos cardiovasculares ${ }^{26}$. Por ello especialistas recomiendan realizar ultrasonografía carotídea como parte del estudio del paciente hipertenso, con el objetivo de intervenir precozmente en la progresión de la enfermedad ateroesclerótica ${ }^{27}$. En relación a esto último es importante comentar que la probabilidad de cursar con un evento cardiovascular dependerá de la carga ateroesclerótica del individuo a lo largo de su vida, la cual puede ser determinada con imágenes como se advierte en un reciente estudio ${ }^{28}$.

En este estudio la población fue mayoritariamente calificada de bajo riesgo por PRF-AdCh, a pesar de una considerable carga de factores 
de riesgo. Este resultado no fue una sorpresa, de hecho, Icaza y cols. mencionan que la mayor distribución de la puntuación chilena es a bajo riesgo, especialmente en mujeres, basado en el análisis de menor mortalidad por cardiopatía isquémica en Chile versus Estados Unidos ${ }^{16}$. Esto es comprensible considerando la estadística nacional, sin embargo, falta validarlo prospectivamente en una cohorte chilena.

En nuestro estudio 14\% de los sujetos de bajo riesgo por PRF-AdCh presentó placa, siendo más prevalente en mujeres. Esta información permitiría reclasificar al sujeto a una categoría de riesgo mayor, indicando tratamiento más intensivo de sus factores de riesgo. Lo desconcertante es que sólo se recomienda solicitar estudio de imágenes a sujetos de riesgo moderado.

Esta situación no sólo se limita a la PRF-AdCh sino también ha sido descrita con Framingham tradicional y ultrasonido carotídeo en población caucásica ${ }^{29,30}$. Así, Navqi y cols. describen presencia de placa en más de $50 \%$ de los sujetos de riesgo bajo ${ }^{31}$. Otro estudio en mexicanos residentes en Estados Unidos detectó en 39\% de sujetos de bajo riesgo, aterosclerosis subclínica a la ultrasonografía carotídea ${ }^{32}$. En población latinoamericana, Christen y cols. detectaron $53 \%$ de placas en argentinos asintomáticos de bajo riesgo por ATPIII ${ }^{33}$. La explicación de la estimación de bajo riesgo pudiese ser que estas puntuaciones evalúan eventos coronarios duros, al igual que la PRF-AdCh.

Parece razonable evaluar con marcadores que optimicen la estratificación, fundamentalmente en la mujer. En este estudio fueron las mujeres las más subestimadas. Este hallazgo se repite en la literatura no sólo con ultrasonografía carotídea sino también con calcio coronario. Michos y cols. reportaron que, en mujeres estudiadas con calcio coronario, $84 \%$ de las con calcio coronario elevado, sobre percentil 75 , calificaban en riesgo bajo por $\mathrm{PRF}^{34}$. Frente a esta inadecuada valoración del riesgo en la mujer es necesario buscar herramientas accesibles para el clínico, y de costo razonable. Los biomarcadores inflamatorios tales como la proteína $\mathrm{C}$ reactiva ultrasensible son de utilidad, sin embargo, en la mujer pueden estar elevados por el uso de estrógenos y por patología reumatológica más prevalente en éstas ${ }^{35}$. Es por eso que apoyamos la observación de Eleid y cols., que la ultrasonografía carotídea puede tener un rol en la identificación de mujeres de mayor riesgo cardiovascular ${ }^{30}$.

En este estudio, la PRF-CVG estratificó la totalidad de la población en un abanico de riesgo, de bajo a alto; logrando a su vez clasificar y discriminar a los de riesgo bajo por PRF-AdCh. En el grupo de bajo riesgo por PRF-CVG, ningún sujeto presentó placa, lo cual contrasta con el Framingham chileno. Esta observación ya fue descrita por Laing y cols., quienes obtuvieron mejor concordancia de la PRF-CVG con presencia de ateroesclerosis subclínica versus PRF tradicional para eventos coronarios mayores ${ }^{32}$. Esta mejor concordancia parece entendible en el marco que la PRF-CVG es más amplia, y le otorga mayor puntaje a la carga de factores de riesgo, puesto que se desea predecir la ocurrencia a 10 años de condiciones cardiovasculares múltiples, tales como ictus y enfermedad vascular periférica. Es por esto que se ha recomendado como puntuación para la atención primaria. La subestimación de riesgo con las puntuaciones son un tema recurrente que ha motivado múltiples reuniones de expertos e incluso se ha considerado ampliar el tiempo de estimación de riesgo cardiovascular a 30 años o a "riesgo de vida" 36 . Lo cual confirma lo confuso del tema.

Apreciamos el trabajo de Icaza y cols., de haber confeccionado una puntuación de riesgo coronario local ${ }^{16}$. Su incorporación en los centros de salud familiar ha sido importante para normar criterios de pesquisa y tratamiento. Sin embargo, se aguarda su validación con eventos duros. También parece necesario ampliar nuestra visión a una prevención cardiovascular inclusiva que incorpore por ejemplo el accidente cerebrovascular. Basado en este estudio, sugerimos en un sujeto con 2 o más factores de riesgo y PRF-AdCh de bajo riesgo, utilizar la PRF-CVG, complementando el estudio con ultrasonido carotídeo sólo en caso de calificar en riesgo moderado con ésta y siempre evaluando costo-efectividad.

Nuestro estudio tiene limitaciones: la cohorte fue pequeña, de un solo centro y no fue seleccionada a través de muestreo epidemiológico. No corresponde a un diseño prospectivo, en búsqueda de futuros eventos cardiovasculares. Además, no se excluyeron pacientes con estatinas.

En conclusión, en población de sujetos chilenos asintomáticos en lo cardiovascular evaluados con dos PRF a 10 años, una de eventos coronarios 
adaptada a Chile y otra de eventos CVG, demostramos quela PRF-AdCh subestima riesgo cardiovascular especialmente en mujeres, siendo menor con la PRF-CVG, y que la adición de imágenes es un aporte en la reclasificación. Estudios con seguimiento de eventos cardiovascular confirmarán cuáles puntuaciones son más válidas.

\section{Referencias}

1. Kannel WB, McGee D, Gordon T. A general cardiovascular risk profile: the Framingham Study. Am J Cardiol 1976; 38: 46-51.

2. Conroy RM, Pyorala K, Fitzgerald AP, Sans S, Menotti A, De Backer G, et al. Estimation of ten-year risk of fatal cardiovascular disease in Europe: the SCORE project. Eur Heart J 2003; 24: 987-1003.

3. Assmann G, Cullen P, Schulte H. Simple scoring scheme for calculating the risk of acute coronary events based on the 10-year follow-up of the prospective cardiovascular Munster (PROCAM) study. Circulation 2002; 105: 310-5.

4. D'Agostino R, Grundy S, Sullivan L, Wilson P. Validation of the Framingham coronary heart disease prediction scores: results of a multiple ethnic groups investigation. JAMA 2001; 286: 180-7.

5. Linton MF, Fazio S. A practical approach to risk assessment to prevent coronary artery disease and its complications. Am J Cardiol 2003; 92 (Suppl): 19i-26i.

6. Perk J, De Backer G, Gohlke H, Graham I, Reiner Z, Verschuren M, et al. European Guidelines on cardiovascular disease prevention in clinical practice (version 2012). Eur Journal of Preventive Cardiology 2012: 19 (4): 585-667.

7. O'Leary D, Bots M. Imaging of atherosclerosis. European Heart Journal 2010; 31: 1682-9.

8. Celermajer DS. Noninvasive detection of atherosclerosis. N Engl J Med 1998; 339: 2014-5.

9. Hodis H, Mack W, La Bree L, Selzer R, Liu Ch, Liu C, et al. The Role of Carotid Arterial Intima-Media Thickness in Predicting Clinical Coronary Events. Arch Int Med 1998; 128: 262-9.

10. O’Leary D, Polack J, Kronmal R, Manolio T, Burke G, Wolfson S. Carotid Artery Intima and Media Thickness as a Risk Factor for Myocardial Infarction and Stroke in Older Adults. N Engl J Med 1999; 340: 14-22.

11. Nguyen-Thanh HT, Benzaquen BS. Screening for subclinical coronary artery disease measuring carotid intima media thickness. Am J Cardiol 2009; 104 (10): 1383-8.

12. Cobble M, Bale B. Carotid intima-media thickness: knowledge and application to everyday practice. Postgrad Med 2010; 122 (1): 10-8.

13. Nambi V, Chambless L, Folsom A, He M, Hu Y, Mosley $\mathrm{T}$, et al. Carotid Intima-Media Thickness and Presence or Absence of Plaque Improves Prediction of Coronary Heart Disease Risk. The ARIC Study. JACC 2010; 55: 1600-7.

14. Polak J, Pencina M, Pencina K, O’Donnell C, Wolf P, D’Agostino R. Carotid -Wall Intima- Media Thickness and Cardiovascular Events. N Engl J Med 2011; 365: 213-21.

15. D'Agostino, Vasan R, Pencina M, Wolf P, Cobain M, Massaro J, et al. General Cardiovascular Risk Profile for Use in Primary Care. The Framingham Heart Study. Circulation 2008; 117: 743-53.

16. Icaza G, Núñez L, Marrugat J, Mujica V, Escobar M, Jiménez A, et al. Estimación de riesgo de enfermedad coronaria mediante la función de Framingham adaptada para la población chilena. Rev Med Chile 2009; 137: 1273-82.

17. Chobanian A, Bakris G, Black H, Cushman W, Green L, Izzo J, et al. The Seventh Report of the Joint National Committee on Prevention, Detection, Evaluation, and Treatment of High Blood Pressure. JAMA 2003; 289: 2560-72.

18. Grundy S, Cleeman J, Daniels S, Donato K, Eckel R, Franklin B, et al. Diagnosis and Management of the Metabolic, Syndrome: An American Heart Association/ National Heart, Lung, and Blood Institute Scientific Statement (Executive Summary). Circulation 2005; 112: 2735-52.

19. www.riesgo_coronario-PIFRECV-Universidad de Talca. http://pifrecv.utalca.cl/htm/riesgo_coronario/utilitarios. htm

20. www.framinghamheartstudy.org/risk/gencardio.html

21. Touboul PJ, Hennerici MG, Meairs S, Adams H, Amarenco P, Bornstein N, et al. Mannheim carotid intima-media thickness consensus (2004-2006). An update on behalf of the Advisory Board of the 3rd and 4th Watching the Risk Symposium, 13th and 15th European Stroke Conferences, Mannheim, Germany, 2004, and Brussels, Belgium, 2006. Cerebrovasc Dis 2007; 23: 75-80.

22. Stein J, Korcarz C, Hurst T, Lonn E, Kendall C, Mohler E, et al. Use of Carotid Ultrasound to Identify Subclinical Vascular Disease and Evaluate Cardiovascular Disease Risk: A Consensus Statement from the American Society of Echocardiography Carotid Intima-Media Thickness Task Force Endorsed by the Society for Vascular Medicine. J Am Soc Ecocar 2008; 21: 93-111.

23. Toubul P, Vicaut E, Labreuche J, Belliard JP, Cohen S, Kownator S, et al. Design, Baseline Characteristics and 
Carotid Intima-Media Thickness Reproducibility in the PARC Study. Cerebrovasc Dis 2005; 19: 57-63.

24. Toubul P, Vicaut E, Labreuche J, Acevedo M, Torres V, Ramírez-Martínez J, et al. Common Carotid Artery Intima Media-Thickness: The Cardiovascular Risk Factor Multiple Evaluation in Latin America (CARMELA) Study Results. Cerebrovasc Dis 2011; 31: 43-50.

25. R Core Team (2013). R: A language and environment for statistical computing. R Foundation for Statistical Computing, Vienna, Austria. URL http://www.R-project.org/.

26. Davidsson L, Fagerberg B, Bergstróm G, Schmidt C. Ultrasound-assessed plaque occurrence in the carotid and femoral arteries are independent predictors of cardiovascular events in middle aged men during 10 years of follow-up. Atherosclerosis 2010; 209: 469-73.

27. Mancia G, Fagard R, Narkiewicz K, Redon J, Zanchetti A, Böhm M, et al. ESH/ESC Guidelines for the management of arterial hypertension. European Heart Journal 2013; 34: 2159-219.

28. Baber U, Mehran R, Sartori S, Malby Schoos M, Sillesen $\mathrm{H}$, Fuster V, et al. Prevalence, Impact and Predictive Value of Detecting Subclinical Coronary and Carotid Atherosclerosis in Asymptomatic Adults. The Bio Image Study. J Am Coll Cardiol 2015; 65 (11): 1065-74.

29. Wilson PW, D'Agostino RB, Levy D, Belanger AM, Silbershatz H, Kannel WB. Prediction of coronary heart disease using risk factor categories Circulation 1998; 97 : 1837-44.

30. Eleid M, Lester S, Wiedenbeck T, Patel S, Appleton C, Nelson M, et al. Carotid Ultrasound Identifies High
Risk Subclinical Atherosclerosis in Adults with Low Framingham Risk Scores. J Am Soc Echocardiogr 2010; 23: 802-8.

31. Naqvi T, Mendoza F, Raffi F, Gransar H, Guerra M, Lepor N, et al. High Prevalence of Ultrasound Detected Carotid Atherosclerosis in Subjects with Low Framingham Risk Score: Potential Implications for Screening for Subclinical Atherosclerosis. J Am Soc Echocardiogr 2010; 23: 802-8.

32. Laing S, Smulevitz B, Vatcheva K, Rentfro A, McPherson D, Fisher-Hoch S, et al. High Prevalence of Subclinical Atherosclerosis by Carotid Ultrasound among Mexican Americans: Discordance with 10-Year Risk Assessment using the Framingham Risk Score. Echocardiography 2012; 29: 1224-32.

33. Christen A, Elikir G, Brandani L, Miranda A, Graf S, Ramírez A, et al. Ateroesclerosis subclinical y estimación de riesgo coronario: comparación de tablas de riesgo. Rev Argent Cardiol 2006; 74: 433-40.

34. Michos E, Nasir K, Braunstein J, Rumberger J, Buddof $\mathrm{M}$, Blumental R, et al. Framingham risk equation underestimates subclinical atherosclerosis in asymptomatic women. Atheoesclerosis 2006; 184: 201-6.

35. Ricker P, Hennekens C, Rifai N, Buring J, Manson J. Hormone replacement therapy and increased plasma concentration of C-Reactive Protein. Circulation 1999; 100: 713-6.

36. Lloyd Jones D, Wilson P, Larson M, Leip E, Beiser A, D'Agostino R, et al. Lifetime risk of coronary heart disease by cholesterol levels at selected ages. Arch Intern Med 2009; 203; 163: 1966-72. 